\title{
Choosing the Best Glasses for Clinical Practice: Evidence- Based Medicine Versus Other Alternative Approaches
}

\author{
Masoud Saberi ${ }^{1, *}$ \\ ${ }^{1}$ Department of Anesthesiology, Kasra Hospital, Tehran, Iran \\ "Corresponding author: Masoud Saberi, Department of Anesthesiology, Kasra Hospital, Tehran, Iran. Tel: +98-9123763746, E-mail: mas_sa_sh@yahoo.com
}

Received 2016 December 01; Accepted 2016 December 19.

Keywords: Evidence-Based Medicine, Medicine

Do you belong to the camp of the physicians who wear glasses at work to have a more accurate eyesight? Do you hold the opinion that anyone who does not wear glasses is somewhat categorized as one who does not subscribe to the idea that wearing glasses gives him a sharper view of the world and puts him in a better position to pass judgments?

The fact of the matter is that a physician, besides wearing physical eyeglasses, may an advantage of other mechanisms that function as glasses for him to diagnose the overt and covert symptoms for a variety of diseases and ailments, deeply analyze them, and decide on a prognosis course for each patient accordingly. Students of medicine and their patients habitually carry such metaphorical glasses, and every single one of them gets used to that particular set of lenses over time, resulting in viewing the treatment with their own set of beliefs and biases.

The set of lenses that reflect the reality in a faithful and accurate way are the ones that are true to evidence-based medicine, while other lenses undermine or overestimate the facts at hand and result in an ultimately distorted view of the situation. Currently, for instance, every single decision taken by physicians is under strict scrutiny by the public. Hence, it is not surprising that the medical community and its elite members may look at their patients through the lenses biased with law and liability issues in medicine.

Perhaps it would be fair to state that the most frequently used set of lenses by a physician are the ones that reflect eminence-based medicine. The physician might have made certain medical decisions without ever documenting the cases, so they could not be analyzed in a accurate manner. The more experience the physician gains over years of practicing medicine, the more he subscribes to this point of view and the less he values gathering the evidences that are based on the real cases. Year after year, the physician makes critical decisions for different cases by gathering the symptoms selectively based on analyzing the ones that are compatible with his biased opinion, while these decisions could be fundamentally wrong with a faulty patient prognosis management. Such highly respected physicians are typically the ones who are pictured with white or receding hair line and a halo of glory around their heads. This set of lenses move contagiously from one physician to the next, so a young physician who looks up to this type of practitioner will follow the same path subconsciously, implementing these faulty diagnoses over again.

A physician may occasionally ignore all the medical evidence, leading him and the onlookers to a wrong path and blocking the possibility of any second opinion. Vehemence-based medicine can be a metaphorical description of such situations. Many medical practitioners consider a pivotal role for God or the nature in the course of treatment, or leave the course of events to a sealed destiny from time to time, which can be labeled as wearing providence-based medicine lenses. This viewpoint can be beneficial as long as it provides the very much needed comfort and soothing for the patient. Nonetheless, if it results in ignoring the patient and seizing to implement the much-needed medical care, it may have disastrous followups. More seriously, at a global scale, if it leads to complacency and not striving to improve the quality of medical services, the prospects look very bleak indeed. For instance, the medical community could adopt a complacent view upon reaching the surprisingly low rate of 1 in 20000 for the patients under anesthesia many years back. However, if they looked upon this number of fatalities as God's will, the rate would have never dropped to an even lower record of 1 in 180000 after years of endeavor and heavy investment.

You may have noticed a dashing young medical professor who was eloquently dressed including a top brand tailored pair of suits, a gilded watch, and a heavy gold necklace, a silk tie along with a paced and friendly tone. These are all typical features of an attitude referred to as eloquence-based medicine.

A novice or less proficient physician or the one who suf- 
fers from a worrisome or unstable character when treating patients with unconventional symptoms in a situation with frequent liability cases, may choose a line of action that leads to frequent lab tests requests and radical courses of treatment with frequent changes. This is a typical case of nervousness-based medicine.

There is yet another set of lenses worn only by the surgeons. Relying on their established sense of self- confidence, they like to do whatever they can for their patients during the course of the surgery. This is due to the fact that their fixed lenses of confidence-based medicine drives them towards this felling of doing something very effective for the patients. In sharp contrast with the previous case, physicians with firm conviction in diffidence-based medicine may believe they know the right course of action, but lack the confidence to act upon their beliefs $(1,2)$.

Considering the global atmosphere of the medical community, all physicians try to wear the lenses for the evidence-based medicine as far as the research findings make it convenient for them. Yet, is this the end to the scenario of medical choices for the physicians? I do not believe so.

In the course of treatment, the emotional aspect and the existence of a firm bond between the patients and those who accompany them is of utmost significance. If the physician is deeply familiar with other aspects of the patient's life, he can then choose between the courses of action that are more in tune with the patient's psychological condition and cultural and family background. Evidencebased medicine provides us with the most comprehensive stance, but it may alienate us from the psychological side. So, it seems reasonable to try wearing a set of lenses based on our local culture, which can be labeled as humanity-based medicine. In this procedure, the physician first tries to decide on the right course of treatment based on evidence-based medicine, and then modifies it based on the patient's emotional, cultural, and social conditions to make the best decision to support the patient.

In any field of science including medicine and engineering, one should initially master the technical skills of his profession that are interpreted as evidence-based medicine for a physician. Besides this indispensable aspect, the leaders of the field are also equipped with another equally effective and significant strategy, which might be referred to as non-technical skill. This can be interpreted as using the resources at hand including the medical staff and facilities in the most efficient way to achieve the optimum results. These 2 aspects of being armed with both technical and non-technical skills are relevant in all fields of science, but it may be stated that the human side of the story is of utmost importance in the field of medicine. The fact is that a physician involves all his emotions and em- pathizes with the patient to join him in his totality with all his feelings, life-story, and unique manners. The physician, then, uses his technical skills or position based on evidence-based medicine and his non-technical skills to form communication talents to treat his patient.

\section{References}

1. Isaacs D, Fitzgerald D. Seven alternatives to evidence based medicine. BMJ. 1999;319(7225):1618. [PubMed:10600968].

2. Sharar DA. Evidence-based practice in managed care: more pro paganda than reality? Behav Healthc Tomorrow. 2003;12(1):SR22-4. [PubMed: 12619605]. 